\title{
Commentary: Charting the path to a functional bioengineered heart
}

\author{
Amine Mazine, MD, and Vivek Rao, MD, PhD
}

\author{
From the Division of Cardiovascular Surgery, Peter Munk Cardiac Centre, Toronto General Hospital, University \\ of Toronto, Toronto, Ontario, Canada. \\ Disclosures: Authors have nothing to disclose with regard to commercial support. \\ Received for publication July 13, 2019; accepted for publication July 17, 2019; available ahead of print Aug 14, \\ 2019. \\ Address for reprints: Vivek Rao, MD, PhD, Cardiovascular Surgery, Peter Munk Cardiac Centre, Toronto General \\ Hospital, 200 Elizabeth St, Toronto, ON M5G 2C4, Canada (E-mail: vivek.rao@uhn.ca). \\ J Thorac Cardiovasc Surg 2020;159:1361-2 \\ $0022-5223 / \$ 36.00$ \\ Copyright (C) 2019 by The American Association for Thoracic Surgery \\ https://doi.org/10.1016/j.jtcvs.2019.07.042
}

In this issue of The Journal of Thoracic and Cardiovascular Surgery, Taylor and colleagues discuss recent advances and ongoing challenges in building a human-sized, functional bioengineered heart. ${ }^{1}$ Their article focuses on 2 recently published studies that incorporated induced pluripotent stem cell induced pluripotent stem cell-derived cardiomyocytes into biomimetic scaffolds consisting of either electrospun nanoscaffolds ${ }^{2}$ or 3 -D printed constructs. ${ }^{3}$ Although Taylor and colleagues state that these studies represent a step forward and an exciting proof of concept, they also highlight some of the important limitations of these approaches. Such limitations include absence of appropriate vascularization, omission of important noncardiomyocyte cell types, and lack of functional capability.

As a comparison, the authors also succinctly describe their own approach of cadaveric organ perfusion decellularization-introduced more than a decade ago ${ }^{4}$ — and highlight the advantages of this approach in generating a scaffold for total heart bioengineering. These advantages include the ability to recapitulate the complex 4-chamber architecture of the normal heart, appropriate vascularization and the ability to provide repopulating cells with critical biochemical and biophysical cues. However, the authors do not delve into the limitations and unaddressed questions surrounding the perfusion-decellularization approach, such as issues of biocompatibility and appropriate sympathetic/ parasympathetic responses to neural stimuli.

Perhaps the most important limitation of the perfusion decellularization approach is the fact that this strategy has not been successfully applied to large and complex prototypes. Indeed, although several reports have shown that recellularization of small scaffolds with cardiomyocytes can lead to adequate cell alignment, contraction, and electrical integration, no study has shown adequate function on a clinically relevant scale. Taylor and colleagues identify lack of viable recellularization strategies as the major road block in this endeavor. Indeed, although most of the discussion in this article focuses on the optimal way to generate a

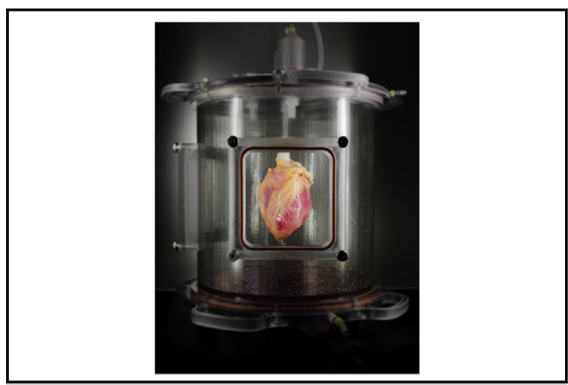

Decellularized human whole-heart scaffold, reseeded with induced pluripotent stem cell-derived cardiomyocytes. Courtesy of Bernhard Jank, Harald Ott Laboratory, Center for Organ Engineering, Massachusetts General Hospital, Harvard Medical School.

\section{Central Message}

Bioengineering a functional, human-sized heart will require strategies for the efficient, reproducible, and cost-effective cellularization of biomimetic scaffolds.

See Article page 1357.

biomimetic scaffold, the authors astutely point out that complete and appropriate recellularization of such a scaffold remains the most important challenge facing scientists in this field. In particular, cell manufacturing processes that allow for the efficient, reproducible and cost-effective production of large number of appropriate cells are lacking.

Beyond the reliable and scalable production of the various cardiac cell types, another foreseeable challenge will be the integration of these cells into a scaffold. Delivery methods that allow the precise distribution of these cells in their anatomically correct location have yet to be developed. Furthermore, it is increasingly recognized that complex cell-cell interactions play a critical role in homeostasis. So far, this interplay between different cardiac cell types has primarily been studied in 2-dimensional culture systems, as well as organoids. ${ }^{5}$ Whether these interactions can be recapitulated within a human-sized bioengineered scaffold remains to be seen.

The quest to build a replacement for the failing human heart has been going on for more than half a century. Advances in transplantation and total artificial heart therapies have transformed the care of heart failure, but these approaches have significant limitations (eg, limited donor availability, need for immunosuppression or anticoagulation, externalized power source, etc). Bioengineering a 
functional, human-sized heart would represent the next quantum step in the treatment of heart failure. In light of the aforementioned challenges, this appears to be a farfetched objective. Nonetheless, the use of scaffold-based technologies to repair or replace parts of the heart (eg, myocardial infarction scar, diseased valve) is a much more realistic undertaking and one that could materialize in the near future. ${ }^{6}$

\section{References}

1. Taylor DA, Hochman-Mendez C, Sampaio LC. Are we close to bioengineering a human-sized, functional heart? J Thorac Cardiovasc Surg. 2020;159:1357-60.
2. MacQueen LA, Sheehy SP, Chantre CO, Zimmerman JF, Pasqualini FS, Liu X, et al. A tissue-engineered scale model of the heart ventricle. Nat Biomed Eng. 2018;2:930-41.

3. Noor N, Shapira A, Edri R, Gal I, Wertheim L, Dvir T. 3D printing of personalized thick and perfusable cardiac patches and hearts. Adv Sci (Weinh). 2019;6:1900344.

4. Ott HC, Matthiesen TS, Goh SK, Black LD, Kren SM, Netoff TI, et al. Perfusiondecellularized matrix: using nature's platform to engineer a bioartificial heart. Nat Med. 2008;14:213-21.

5. Nugraha B, Buono MF, von Boehmer L, Hoerstrup SP, Emmert MY. Human cardiac organoids for disease modeling. Clin Pharmacol Ther. 2019;105: 79-85.

6. Montgomery M, Ahadian S, Davenport Huyer L, Lo Rito M, Civitarese RA, Vanderlaan RD, et al. Flexible shape-memory scaffold for minimally invasive delivery of functional tissues. Nat Mater. 2017;16:1038-46. 